\title{
QPS - Modelo para Avaliação da Qualidade de Produtos de Software: Resultados Iniciais
}

\author{
Ana Regina da Rocha ${ }^{1}$, Guilherme Horta Travassos ${ }^{1}$, \\ Gleison Santos ${ }^{2}$, Sheila Reinehr ${ }^{3}$ \\ ${ }^{1}$ COPPE/UFRJ - Universidade Federal do Rio de Janeiro - Caixa Postal 68511 - \\ CEP 21.941-972 - Rio de Janeiro, RJ \\ 2 Programa de Pós-Graduação em Informática - Universidade Federal do Estado do Rio \\ de Janeiro (UNIRIO) - Av. Pasteur, 458, Urca, CEP 22290-240 - Rio de Janeiro, RJ \\ ${ }^{3}$ Programa de Pós-Graduação em Informática - Pontifícia Universidade Católica do \\ Paraná (PUC-PR), R. Imaculada Conceição, 1155 - CEP 80215-901 - Curitiba, PR \\ \{darocha, ght\}@cos.ufrj.br, gleison.santos@uniriotec.br, \\ sheila.reinehrepucpr.br
}

\begin{abstract}
The quality of a software product should be seen in a broader manner and take into account four important dimensions: Software Engineering, Service, Product Quality and Organizational. Standards and maturity models address elements of these dimensions but none of them consider all of them to evaluate software products in a broad and holistic way. That leads organizations to use different models to evaluate their procedural and organizational diversity. In this paper, we present the QPS model, which allows the evaluation of software products quality, and the first results of its implementation and assessments in Brazilian organizations.
\end{abstract}

Resumo. A qualidade de um produto de software deve ser vista de forma ampla, considerando-se quatro dimensões importantes: Engenharia de Software, Serviço, Qualidade do Produto e Organizacional. Apesar de existirem normas e modelos que tratem elementos destas dimensões, não há nenhum modelo que avalie produtos de software considerando estas quatro dimensões de uma forma ampla e holística, levando as organizações a lançarem mão de diferentes modelos para garantirem a avaliação de sua diversidade procedimental e organizacional. Este artigo apresenta o modelo QPS, um modelo para avaliação da qualidade de produtos de software, e os primeiros resultados de sua implementação e avaliação em organizações brasileiras.

\section{Introdução}

A qualidade de um produto de software deve ser vista de forma ampla, levando-se em consideração as dimensões: organizacional, engenharia de software, serviço e produto. Estas dimensões se justificam considerando, por exemplo, os seguintes cenários: (1) Dimensão Organizacional - uma empresa com um produto no mercado precisa ter uma documentação do produto, processos organizacionais e atributos de processo que garantam a implementação desta dimensão e das dimensões de Engenharia de Software e de Serviços; (2) Dimensão de Engenharia de Software - uma empresa com um produto no mercado precisa ter processos de manutenção corretiva, evolutiva e 
adaptativa (quando pertinente); (3) Dimensão de Serviço - uma empresa com um produto no mercado precisa ter um serviço de atendimento ao cliente; e, (4) Dimensão de Qualidade do Produto - um produto de software deve possuir uma descrição do produto, uma documentação para o usuário e características de qualidade que podem ser: (i) características essenciais a qualquer produto; (ii) características que devem estar presentes em determinados produtos para atender às suas especificidades; e, (iii) características de qualidade do produto em uso.

Existem, atualmente, normas e modelos para a definição e avaliação de processos de Engenharia de Software, tais como: a norma internacional ISO/IEC 12207 [ISO/IEC, 2008a] - que define os processos do ciclo de vida de software; a série de normas internacionais ISO/IEC 33000 [ISO/IEC, 2015] - que define níveis de capacidade e forma de avaliá-los; o modelo de maturidade internacional CMMI-DEV [CMMI Product Team, 2010a] - que define processos de software, níveis de maturidade, níveis de capacidade e forma de avaliá-los; e, o modelo de maturidade nacional MRMPS-SW [SOFTEX, 2016] - que define processos de software, níveis de maturidade e forma de avaliá-los.

Existem, também, normas e modelos para avaliação de processos de serviços, tais como: a norma internacional ISO/IEC 20000 [ISO/IEC, 2011] - que define processos de serviços; o modelo de maturidade internacional CMMI-SVC [CMMI Product Team, 2010] - que define processos de serviços, níveis de maturidade, níveis de capacidade e forma de avaliá-los; e, o modelo de maturidade nacional MR-MPS-SV [SOFTEX, 2015] - que define processos de serviços, níveis de maturidade, níveis de capacidade e forma de avaliá-los.

No que se refere à dimensão de qualidade do produto, existe a série de normas internacionais ISO/IEC 25000 [ISO/IEC 2014].

Não existe modelo algum que avalie produtos de software, considerando as quatro dimensões citadas, de uma forma ampla e holística, o que leva as organizações a lançarem mão de diferentes frentes de trabalho, iniciativas e investimentos para garantir a avaliação de sua diversidade procedimental e organizacional. O Modelo para Avaliação da Qualidade de Produtos de Software (QPS) tem o objetivo de suprir esta carência. Este artigo apresenta o modelo QPS e os primeiros resultados de sua implementação e avaliação em organizações brasileiras.

Além desta seção introdutória, o artigo está organizado da seguinte forma: Seção 2 apresenta uma breve revisão da literatura; Seção 3 descreve a visão geral do modelo; Seção 4 apresenta os resultados iniciais das avaliações piloto do QPS; por fim, a Seção 5 apresenta as considerações finais.

\section{Referencial Teórico}

\subsection{Modelos e Normas de Processo de Software}

Ao longo dos anos, desde a década de 80 , diversas normas e modelos de referência têm sido desenvolvidos para apoiar a melhoria dos processos de software nas organizações. Dentre as normas, a que mais se destaca é a ISO/IEC 12207 [ISO/IEC, 2008a] que descreve os processos do ciclo de vida de software, de acordo com o relacionamento entre eles e com a responsabilidade pela execução de cada um. Estes processos estão divididos em duas categorias: os processos relacionados ao contexto de sistema e os 
processos relacionados especificamente ao software. $\mathrm{Na}$ primeira categoria se encontram: Processo de Acordo; Processos de Capacitação de Projetos Organizacionais; Processos de Projeto e Processos Técnicos. Na segunda categoria, por sua vez, se encontram: Processos de Implementação de Software; Processos de Apoio de Software; e, Processos de Reúso de Software.

O conjunto de normas da ISO/IEC que se relaciona com a avaliação destes processos é denominado Série ISO/IEC 33000 [ISO/IEC, 2015]. Esta série de normas definem níveis de capacidade que são utilizados na avalição dos processos que estão definidos na ISO/IEC 12207. Basicamente a Norma define os seguintes níveis de capacidade: (0) incompleto; (1) executado; (2) gerenciado; (3) estabelecido; (4) previsível; e, (5) em otimização.

Ainda na década de 80 surgiu um modelo para melhoria de processos que prevê níveis de maturidade, que podem ser compreendidos como degraus na evolução da qualidade no desenvolvimento de software. O principal representante desta abordagem é o CMMI-DEV (Capability Maturity Model Integration - for Development) [CMMI Product Team, 2010]. Ele foi desenvolvido pelo SEI (Software Engineering Institute) e agora está sob a gestão do CMMI Institute (atualmente associado à ISACA). O modelo prevê 5 níveis de maturidade que vão desde o nível 1 (menos maduro) até o nível 5 (mais maduro). Prevê áreas de processo que podem ser agrupadas em 4 categorias: Gerência de Projetos, Gerência de Processos, Engenharia e Apoio. Estas Áreas de Processo são definidas e agrupadas nos níveis de maturidade [CMMI Product Team, 2010].

No Brasil, o programa iniciado pela SOFTEX em 2003, denominado MPS.BR (Melhoria de Processo do Software Brasileiro), introduziu modelos de maturidade para a melhoria de processos em diversas áreas, entre elas, a área de software, com o modelo MR-MPS-SW [SOFTEX, 2016]. Este modelo de referência foi também concebido utilizando o conceito de níveis de maturidade. Sua estrutura prevê 7 níveis evolutivos de maturidade que têm início no Nível G (Parcialmente Gerenciado) e culminam no Nível A (Em otimização). O modelo guarda certa compatibilidade com o modelo CMMI$\mathrm{DEV}$, exceto pela presença de alguns processos adicionais que são provenientes da Norma ISO/IEC 12207 (Gerência de Portfólio de Projetos, Gerência de Reutilização e Desenvolvimento para Reutilização).

\subsection{Modelos e Normas de Gerenciamento de Serviços de TI}

Paralelamente às preocupações com a qualidade dos processos de software, nasceram e se proliferaram também os modelos para o gerenciamento de serviços de TI. Nesta linha, o mais conhecido e utilizado internacionalmente é o ITIL (Information Technology Infrastructure Library). De acordo com a OGC (2011), o modelo provê "um guia para os provedores de serviços para a provisão de serviços de TI de qualidade e para os processos, funções e outras capacidades necessárias para suportá-los.”. Os processos que o compõem estão divididos em 5 categorias: Estratégia do Serviço; Projeto do Serviço; Transição do Serviço; Operação do Serviço; e, Melhoria Contínua do Serviço.

$\mathrm{Na}$ esteira dos modelos de serviços, o SEI lançou também um modelo de maturidade para a área de serviços: CMMI-SVC [CMMI Product Team, 2010b]. Este modelo, originalmente baseado no SW-CMM, também foi estruturado em 5 níveis 
evolutivos de maturidade, iniciando no Nível 1 e culminando no Nível 5. Comparado à utilização do CMMI-DEV, este ainda é um modelo jovem e em fase de consolidação, com um número muito menor de avaliações realizadas.

No Brasil, o programa MPS.BR também introduziu um modelo na área de gerenciamento de serviços de TI, o modelo MR-MPS-SV [SOFTEX, 2015]. Este modelo de referência foi também concebido utilizando o conceito de níveis de maturidade, só que desta vez aplicado a serviços. Sua estrutura prevê 7 níveis evolutivos de maturidade, assim como o seu antecessor da área de software (MR-MPS-SW). Seu início também se dá no Nível G (Parcialmente Gerenciado) e culmina no Nível A (Em otimização). O modelo guarda certa compatibilidade com o modelo CMMI-SVC, porém com interpretações mais claras sobre alguns dos conceitos de serviços.

A Norma Internacional que trata do gerenciamento de serviços de TI é a ISO/IEC 20.000 [ISO/IEC, 2011; 2012]. Esta Norma possui uma correlação íntima com o modelo de referência ITIL. Seu foco é a melhoria dos processos para a área de serviços de TI. Trata-se, na realidade, de uma família de normas, composta por diversas partes. Na parte principal está definida a estrutura da Norma que foca grupos de processos que estão relacionados a Entrega de Serviços, Controle, Liberação, Resolução e Relacionamento.

\subsection{Norma para a Qualidade de Produto de Software}

A principal Norma Internacional que trata da Qualidade do Produto de Software é a ISO/IEC 25000 [ISO/IEC, 2014]. A série, também conhecida como SQuaRE, possui três modelos de qualidade: qualidade em uso, qualidade de produto e qualidade de dados. Cada um destes modelos é composto por características e sub-características de qualidade que podem ser usadas em todo ou em parte, e acordo com a visão de cada stakeholder. O modelo de Qualidade em Uso possui 5 características: Efetividade, Eficiência, Satisfação, Liberdade de Risco e Cobertura de Contexto. O modelo de qualidade de produto possui 8 características: Adequação Funcional, Eficiência de Desempenho, Compatibilidade, Usabilidade, Confiabilidade, Segurança, Manutenibilidade e Portabilidade. O modelo de Qualidade de Dados possui 15 características que podem ser encontradas em ISO/IEC 25012 [ISO/IEC, 2008b].

\section{Visão Geral do Modelo QPS}

Tendo em vista a amplitude da avaliação e a necessidade de se manter em conformidade com as perspectivas atuais da qualidade, a dimensão de Engenharia de Software e a Dimensão Organizacional têm por base as Normas Internacionais ISO/IEC 12207 [ISO/IEC, 2008a] e ISO/IEC 33020 [ISO/IEC, 2015]. A dimensão de Serviços tem por base as Normas Internacionais ISO/IEC 20000 [ISO/IEC, 2011] e ISO/IEC 33000 [ISO/IEC, 2015]. A dimensão de características de qualidade do produto tem por base a série de Normas Internacionais ISO/IEC 25000 [ISO/IEC 2014]. O processo de avaliação está baseado nas séries de Normas Internacionais ISO/IEC 33000 [ISO/IEC, 2015] e ISO/IEC 25000 [ISO/IEC 2014]. A Figura 1 apresenta as dimensões do modelo QPS. 


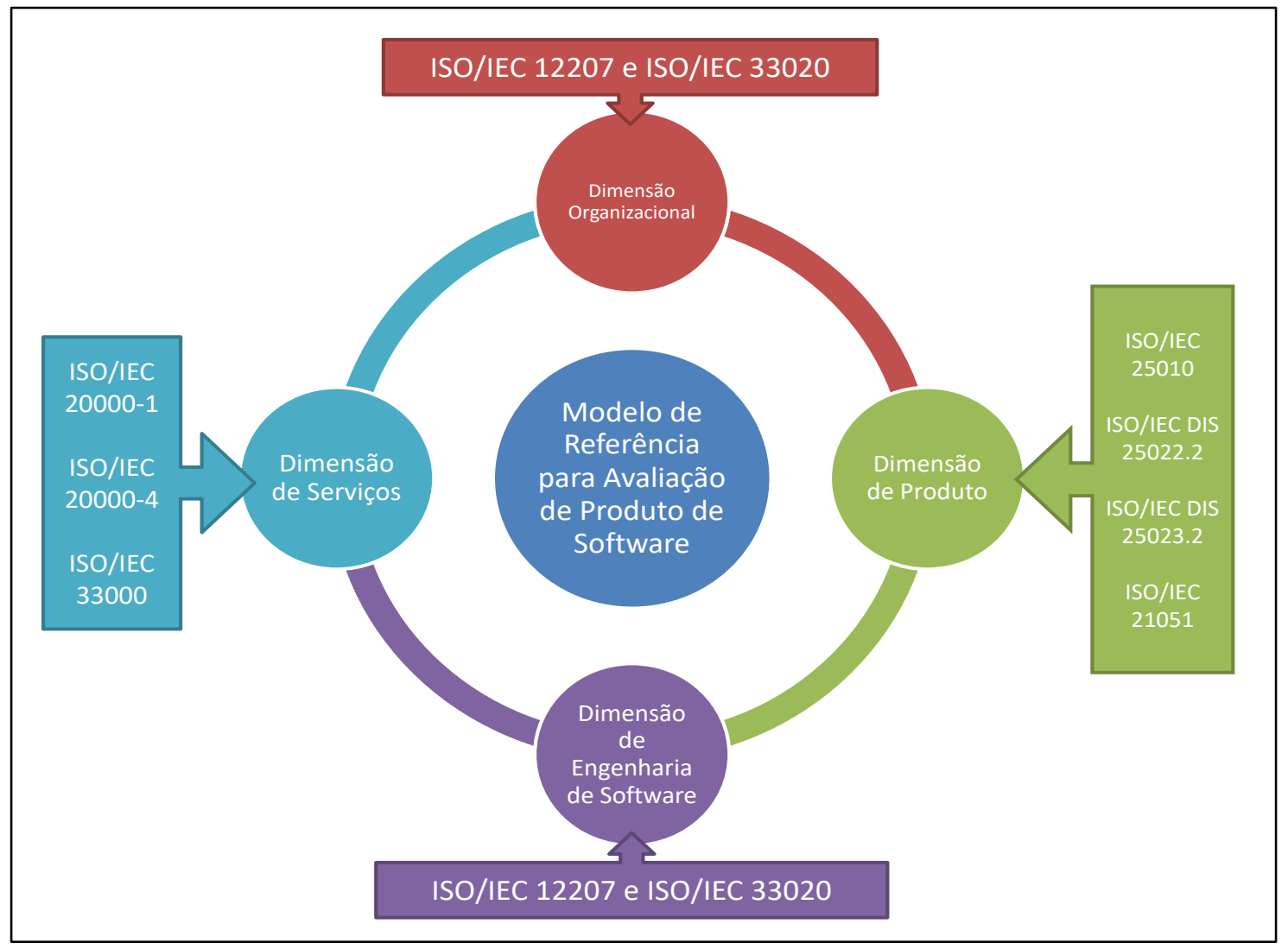

Figura 1 - Vis ão do Modelo QPS para Avaliação de Produtos de Software e sua relação com Normas Internacionais.

O modelo QPS (QPS, 2016) aplica-se a qualquer tipo de produto de software e tem por base os princípios de avaliação contínua, apresentando os resultados em três níveis de atendimento da qualidade: Bronze, Prata e Ouro. Os níveis são acumulativos e, desta forma, o nível Prata inclui todos os requisitos do nível Bronze e o nível Ouro todos os requisitos dos níveis Bronze e Prata.

Durante uma avaliação do modelo QPS são consideradas, de forma diferenciada, duas situações:

1. Avaliação de um produto já disponível e em uso pelo mercado.

2. Avaliação de um produto no seu lançamento, visando a que seja lançado com uma avaliação.

As subseções a seguir descrevem as duas formas de avaliação do modelo QPS além do seu processo de avaliação.

\subsection{Avaliação de um produto já disponível e em uso pelo mercado}

A avaliação de um produto já disponível e em uso no mercado considera:

i. Na Dimensão Organizacional, a documentação existente do produto e a definição e execução de processos relacionados à gestão do produto; 
ii. Na Dimensão de Engenharia de Software, os processos definidos e executados para manutenções corretivas, evolutivas e adaptativas (customizações para diferentes clientes, se pertinente)

iii. Na Dimensão de Serviços, os processos definidos e executados para o fornecimento do produto e o atendimento ao cliente;

iv. Na Dimensão de Qualidade do Produto, a descrição do produto, a documentação do usuário, as características de qualidade gerais e específicas pertinentes ao produto e a qualidade em uso observada pelos usuários.

A avaliação de um produto já disponível no mercado avalia, portanto, a qualidade do produto e como a empresa fornecedora do produto faz a gestão do produto e vem realizando as manutenções, o fornecimento do produto e o atendimento ao cliente. Esta avaliação tem validade de três anos.

$\mathrm{Na}$ dimensão Organizacional são considerados, na avaliação, os documentos relativos a requisitos do produto, rastreabilidade dos requisitos, objetivos de negócio para o produto, descrição da estrutura para fornecimento do produto e atendimento ao cliente, riscos identificados para o produto, melhorias identificadas para o produto, arquitetura do produto e casos de teste para o produto. São também avaliadas: a descrição e implementação dos processos aquisição (quando pertinente), a gerência de configuração do produto e a medição. Para produtos já disponíveis no mercado, é possível avaliar a execução dos processos, portanto, são avaliados atributos de processo de acordo com a ISO/IEC 33.020 [ISO/IEC, 2015]. O Quadro 1 mostra as exigências da dimensão organizacional para cada um dos níveis do modelo QPS.

Quadro 1 - Requis itos da dimensão Organizacional do Modelo QPS.

\begin{tabular}{|c|c|c|c|}
\hline \multirow{2}{*}{ DIMENSÃO ORGANIZACIONAL } & \multicolumn{3}{|c|}{ Nível } \\
\hline & Bronze & Prata & Ouro \\
\hline Requisitos do Produto & $\mathrm{X}$ & $\mathrm{X}$ & $\mathrm{X}$ \\
\hline Rastreabilidade Requisitos X Requisitos & $\mathrm{X}$ & $\mathrm{X}$ & $\mathrm{X}$ \\
\hline Rastreabilidade Requisitos X Código & $\mathrm{X}$ & $\mathrm{X}$ & $\mathrm{X}$ \\
\hline Rastreabilidade Requisitos X Casos de Teste & & $\mathrm{X}$ & $\mathrm{X}$ \\
\hline Objetivos de negócio para o produto & $\mathrm{X}$ & $\mathrm{X}$ & $\mathrm{X}$ \\
\hline Estrutura para fornecimento do produto e atendimento ao cliente & $\mathrm{X}$ & $\mathrm{X}$ & $\mathrm{X}$ \\
\hline Riscos do produto & & $\mathrm{X}$ & $\mathrm{X}$ \\
\hline Oportunidades de melhoria identificadas para o produto & $\mathrm{X}$ & $\mathrm{X}$ & $\mathrm{X}$ \\
\hline Arquitetura do Produto & & $\mathrm{X}$ & $\mathrm{X}$ \\
\hline Casos de Teste do Produto & & $\mathrm{X}$ & $\mathrm{X}$ \\
\hline Definição e execução do processo Aquisição (se pertinente) & $\mathrm{X}$ & $\mathrm{X}$ & $\mathrm{X}$ \\
\hline $\begin{array}{l}\text { Definição e execução do processo Gerência de Configuração do } \\
\text { Produto }\end{array}$ & & $\mathrm{X}$ & $\mathrm{X}$ \\
\hline Definição e execução do processo Medição & & & $\mathrm{X}$ \\
\hline Atributo de processo 1.1: O processo é executado & $\mathrm{X}$ & $\mathrm{X}$ & $\mathrm{X}$ \\
\hline Atributo de processo 2.1: A execução do processo é gerenciada & $\mathrm{X}$ & $\mathrm{X}$ & $\mathrm{X}$ \\
\hline $\begin{array}{l}\text { Atributo de processo 2.2: Os produtos de trabalho do processo são } \\
\text { gerenciados }\end{array}$ & & $\mathrm{X}$ & $\mathrm{X}$ \\
\hline Atributo de processo 3.1: O processo é definido & & & $\mathrm{X}$ \\
\hline Atributo de processo 3.2: O processo está implementado & & & $\mathrm{X}$ \\
\hline
\end{tabular}

$\mathrm{Na}$ dimensão Engenharia de Software são avaliados os processos definidos e executados para manutenções corretivas, evolutivas e adaptativas (customizações para diferentes clientes, se pertinente). Nestes processos devem estar integrados os processos 
Gerência de Projetos, Desenvolvimento e Gerência de Requisitos do Projeto, Arquitetura e Projeto, Construção, Validação, Integração do Produto e Liberação do Produto. O Quadro 2 mostra as exigências da dimensão Engenharia de Software para cada um dos níveis do modelo QPS.

Quadro 2 - Requisitos da dimensão Engenharia de Software do Modelo QPS.

\begin{tabular}{|l|c|c|c|}
\hline \multicolumn{1}{|c|}{$\begin{array}{l}\text { DIMENSÃO ENGENHARIA DE SOFTWARE } \\
\text { Definição e execução do processo de manutenção que atenda } \\
\text { aos processos: }\end{array}$} & \multicolumn{3}{|c|}{ Nível } \\
\cline { 2 - 4 } & Bronze & Prata & Ouro \\
\hline Gerência de Projetos & $\mathrm{X}$ & $\mathrm{X}$ & $\mathrm{X}$ \\
\hline Desenvolvimento e Gerência de Requisitos do Projeto & $\mathrm{X}$ & $\mathrm{X}$ & $\mathrm{X}$ \\
\hline Arquitetura e Projeto & & $\mathrm{X}$ & $\mathrm{X}$ \\
\hline Construção & & $\mathrm{X}$ & $\mathrm{X}$ \\
\hline Validação & & $\mathrm{X}$ & $\mathrm{X}$ \\
\hline Integração do Produto & $\mathrm{X}$ & $\mathrm{X}$ & $\mathrm{X}$ \\
\hline Liberação do Produto & $\mathrm{X}$ & $\mathrm{X}$ & $\mathrm{X}$ \\
\hline Atributo de processo 1.1: O processo é executado & $\mathrm{X}$ & $\mathrm{X}$ & $\mathrm{X}$ \\
\hline Atributo de processo 2.1: A execução do processo é gerenciada & & $\mathrm{X}$ & $\mathrm{X}$ \\
\hline $\begin{array}{l}\text { Atributo de processo 2.2: Os produtos de trabalho do processo são } \\
\text { gerenciados }\end{array}$ & & & $\mathrm{X}$ \\
\hline Atributo de processo 3.1: O processo é definido & & & $\mathrm{X}$ \\
\hline Atributo de processo 3.2: O processo está implementado & & \\
\hline
\end{tabular}

Na dimensão de Serviços são avaliados os processos definidos e executados para Planejamento e Monitoração do Serviço, Gerência de Incidentes e de Solicitações de Serviço, Gerência do Nível de Serviço, Gerência de Segurança da Informação (se pertinente), Gerência de Problemas, Orçamento e Contabilização do Serviço, Gerência da Capacidade, Gerência de Mudanças, Gerência de Continuidade e Disponibilidade e Relato de serviços (se pertinente). O Quadro 3 mostra as exigências da dimensão de serviços para cada um dos níveis do modelo QPS.

Quadro 3 - Requisitos da dimensão de Serviços do Modelo QPS.

\begin{tabular}{|l|c|c|c|}
\hline \multicolumn{1}{|c|}{ DIMENSÃO DE SERVIÇOS } & \multicolumn{3}{c|}{ Nível } \\
\cline { 2 - 4 } Definição e execução de processos que atendam: & Bronze & Prata & Ouro \\
\hline Planejamento e Monitoração do Serviço & $\mathrm{X}$ & $\mathrm{X}$ & $\mathrm{X}$ \\
\hline Gerência de Incidentes e de Solicitações de Serviço & $\mathrm{X}$ & $\mathrm{X}$ & $\mathrm{X}$ \\
\hline Gerência do Nível de Serviço & $\mathrm{X}$ & $\mathrm{X}$ & $\mathrm{X}$ \\
\hline Gerência de Segurança da Informação (se pertinente) & $\mathrm{X}$ & $\mathrm{X}$ & $\mathrm{X}$ \\
\hline Gerência de Problemas & & $\mathrm{X}$ & $\mathrm{X}$ \\
\hline Orçamento e Contabilização do Serviço & & $\mathrm{X}$ & $\mathrm{X}$ \\
\hline Gerência da Capacidade & & $\mathrm{X}$ \\
\hline Gerência de Mudanças & & & $\mathrm{X}$ \\
\hline Gerência de Continuidade e Disponibilidade & & & $\mathrm{X}$ \\
\hline Relato de serviços (se pertinente) & $\mathrm{X}$ & $\mathrm{X}$ & $\mathrm{X}$ \\
\hline Atributo de processo 1.1: O processo é executado & $\mathrm{X}$ & $\mathrm{X}$ & $\mathrm{X}$ \\
\hline Atributo de processo 2.1: A execução do processo é gerenciada & & $\mathrm{X}$ & $\mathrm{X}$ \\
\hline $\begin{array}{l}\text { Atributo de processo 2.2: Os produtos de trabalho do processo são } \\
\text { gerenciados }\end{array}$ & & & \\
\hline Atributo de processo 3.1: O processo é definido & & & $\mathrm{X}$ \\
\hline Atributo de processo 3.2: O processo está implementado & & & $\mathrm{X}$ \\
\hline
\end{tabular}

Na dimensão de Qualidade do Produto são considerados, na avaliação, os documentos relativos à descrição do produto e à documentação do usuário. O produto é também avaliado com relação às seguintes características de qualidade: consistência 
operacional, clareza das mensagens, existência de undo, aparência da interface, controle de acesso, apoio da documentação para análise, integridade dos dados, apoio da documentação para testes e disponibilidade de casos de teste.

No nível Prata deve ser evidenciado que foi realizada uma pesquisa de satisfação dos usuários do produto quanto ao grau de atendimento das características de Qualidade em Uso. Deve ser, portanto, evidenciado:

- Que foi realizada a pesquisa de opinião com as empresas usuárias do produto no período máximo de 12 meses antes da data da avaliação;

- Os resultados da pesquisa;

- Que foi realizada a análise dos resultados e foram tomadas decisões para melhoria do produto onde pertinente.

No nível Ouro deve ser evidenciado que foram identificados requisitos de qualidade do produto (específicos de sua natureza), que existem procedimentos para avaliação do grau de atendimento do produto a estes requisitos e que a avaliação foi realizada. Estes requisitos devem ser identificados considerando as características específicas do produto e as necessidades dos diferentes tipos de usuários (por exemplo, usuários finais, usuários indiretos, mantenedores, responsáveis por adicionar conteúdo etc.). Exemplos de características específicas podem ser vistas em Mota e Oliveira (2017) e Silveira e Souza (2017).

O Quadro 4 mostra as exigências da dimensão de qualidade do produto para cada um dos níveis do Modelo QPS.

Quadro 4 - Requis itos da dimensão de Qualidade do Produto do Modelo QPS.

\begin{tabular}{|l|c|c|c|}
\hline \multicolumn{2}{|c|}{ DIMENSÃO DE QUALIDADE DO PRODUTO } & \multicolumn{3}{c|}{ Nível } \\
\cline { 2 - 4 } & Bronze & Prata & Ouro \\
\hline Descrição do Produto & $\mathrm{X}$ & $\mathrm{X}$ & $\mathrm{X}$ \\
\hline Documentação do Usuário & $\mathrm{X}$ & $\mathrm{X}$ & $\mathrm{X}$ \\
\hline Medida: Consistência Operacional & $\mathrm{X}$ & $\mathrm{X}$ & $\mathrm{X}$ \\
\hline Medida: Clareza das Mensagens & $\mathrm{X}$ & $\mathrm{X}$ & $\mathrm{X}$ \\
\hline Medida: Existência de undo & $\mathrm{X}$ & $\mathrm{X}$ & $\mathrm{X}$ \\
\hline Medida: Aparência da Interface & $\mathrm{X}$ & $\mathrm{X}$ & $\mathrm{X}$ \\
\hline Medida: Controle de Acesso & $\mathrm{X}$ & $\mathrm{X}$ & $\mathrm{X}$ \\
\hline Medida: Apoio da Documentação para Análise & $\mathrm{X}$ & $\mathrm{X}$ & $\mathrm{X}$ \\
\hline Medida: Integridade dos Dados & & $\mathrm{X}$ & $\mathrm{X}$ \\
\hline Medida: Apoio da Documentação para Testes & & $\mathrm{X}$ & $\mathrm{X}$ \\
\hline Medida: Disponibilidade de Casos de Teste & & $\mathrm{X}$ & $\mathrm{X}$ \\
\hline Avaliação da qualidade em uso & & $\mathrm{X}$ & $\mathrm{X}$ \\
\hline Definição e avaliação de requisitos de qualidade específicos do produto & & & $\mathrm{X}$ \\
\hline
\end{tabular}

\subsection{Avaliação de um produto no seu lançamento}

A avaliação de um produto no seu lançamento no mercado considera os mesmos documentos e processos avaliados para produtos já disponíveis no mercado. Entretanto, não se considera a implementação dos processos na organização, por não ser pertinente nesta situação. O que se avalia, neste caso, é a qualidade do produto e se a empresa fornecedora do produto está apta a realizar manutenções, atendimento ao cliente e gestão do produto de forma adequada. Não é, também, avaliada a qualidade em uso por não ser pertinente a um produto no momento de seu lançamento. 
Esta avaliação pode ser realizada antes do lançamento do produto no mercado ou no máximo até 3 meses após o lançamento. Tem validade de um ano, contado a partir da data prevista para o lançamento do produto e informada na data da avaliação. A validade de um ano é motivada por não se considerar, neste caso, a implementação dos processos, apenas a definição deles. Após esta data o produto deve ter nova avaliação como produto disponível e em uso pelo mercado, evidenciando de fato a implementação dos processos.

\subsection{Processo de Avaliação}

A avaliação de um produto, segundo o Modelo QPS, é aderente à ISO/IEC 33.000. É uma avaliação contínua com resultado em níveis (Ouro, Prata e Bronze).

A avaliação tem início com um Diagnóstico Inicial onde se avalia o produto de forma completa considerando os requisitos do nível Ouro, ou seja, o nível máximo. Ao final do diagnóstico inicial e considerando seus resultados, a empresa responsável pelo produto escolhe em que nível deseja realizar a avaliação final. Acredita-se que esta abordagem, considerando de início todas as exigências do modelo no Nível Ouro, é adequada porque o diagnóstico inicial fornece um relatório abrangente e de grande valor para a empresa fornecedora do produto, capaz de orientar a melhoria contínua do produto e dos processos.

Participam da equipe de avaliação ao menos dois avaliadores, sendo que um deles desempenha o papel de líder. Não são previstos avaliadores da própria organização, mas a participação de membros da organização é incentivada no diagnóstico inicial.

A avaliação é realizada por uma Entidade Avaliadora credenciada pela Fundação COPPETEC (unidade da COPPE/UFRJ responsável pela interface com empresas). Em uma avaliação, a Entidade Avaliadora e toda a equipe de avaliação devem ser totalmente independentes da organização e do produto avaliado, fornecendo um serviço totalmente independente de $3^{\mathrm{a}}$ parte. Todas as avaliações QPS são auditadas pelo Comitê Diretivo do Modelo QPS que, após aprovação do resultado, emite declaração da avaliação para o produto com o nível obtido.

O modelo QPS tem prevista, em sua estrutura, a existência de Entidades Avaliadoras credenciadas, que devem contar, pelo menos, com uma equipe de dois avaliadores sendo que um deles deve ser avaliador líder. De forma semelhante ao modelo CMMI não estão previstas Entidades Implementadoras e nem implementadores credenciados.

\section{Resultados Iniciais da Implantação e Avaliação do Modelo QPS}

Até o momento foram realizadas três avaliações utilizando o Modelo QPS, sendo que dois produtos já estavam disponíveis no mercado e um estava sendo avaliado no seu lançamento. As três empresas avaliadas possuíam características bem diferentes e foram bem-sucedidas em suas avaliações.

\subsection{WTS Corporate}

Trata-se de um software para gestão de viagens da empresa Monteiro e Gutierrez Sistemas Ltda., do Rio de Janeiro. O produto já se encontra em uso no mercado e foi avaliado com sucesso para o Nível Ouro. 
A empresa responsável pelo produto WTS Corporate já tinha processos modelados e já havia obtido a certificação ISO 9000. Esta empresa contou com uma breve consultoria antes da avaliação QPS em um total de aproximadamente 20 horas. Esta consultoria realizou um gap analysis e orientou a empresa nos ajustes necessários para a realização do Diagnóstico Inicial $\left(1^{\mathrm{a}}\right.$ fase da avaliação QPS). O consultor participou do diagnóstico inicial, apoiando a empresa na resposta aos questionamentos dos avaliadores. Entre o Diagnóstico Inicial e a Avaliação, ele também apoiou a empresa na resolução dos ajustes. O Diagnóstico Inicial foi realizado em setembro de 2016 com duração de 2 dias e a avaliação final um mês após com duração de um dia e meio. A equipe de avaliação foi formada por um avaliador líder e dois avaliadores.

\subsection{Pirâmide}

Trata-se de um software do tipo ERP da empresa PCC Engenharia de Sistemas Ltda. (PROCENGE), de Recife. O produto já se encontra em uso no mercado e foi avaliado com sucesso para o Nível Ouro.

A empresa responsável pelo produto Pirâmide é uma empresa com tradição em melhoria contínua de seus processos. Realizou ao longo dos anos avaliações MPS-SW níveis $\mathrm{G}, \mathrm{F}$ e $\mathrm{C}$, além de uma avaliação CMMI-DEV Nível 2. Entretanto, nunca havia implementado modelo de serviços, nem CMMI-SVC nem MPS-SV. Neste caso, a equipe de avaliação realizou o Diagnóstico Inicial imediatamente após a conclusão de uma avaliação oficial MPS-SW onde a empresa renovou o nível C. Sendo assim, a dimensão de Engenharia de Software e parte da Dimensão Organizacional não foram consideradas na avaliação QPS, por já terem sido avaliadas na avaliação MPS-SW pela mesma equipe. Foi considerado, portanto, neste caso, a avaliação QPS como uma avaliação complementar à avaliação MPS-SW e foram avaliadas apenas as dimensões de Serviços e Qualidade de Produto bem como aspectos da dimensão organizacional não considerados pelo MR-MPS-SW. Esta empresa não teve apoio de consultoria por ter um grupo de processos experiente. O Diagnóstico Inicial foi realizado em dezembro 2016 com duração de 1 dia e a avaliação final em março de 2017 com duração de um dia e meio. A equipe de avaliação foi formada por um avaliador líder e dois avaliadores.

\subsection{RSI}

Trata-se de uma ferramenta de gestão de serviços institucionais da Fundação Oswaldo Cruz (Fiocruz), do Rio de Janeiro, que estava em fase de lançamento para o mercado. Neste caso, após a avaliação de Diagnóstico Inicial a Fiocruz optou por realizar a avaliação final para o nível Bronze e foi bem-sucedida.

A terceira avaliação, realizada na Fiocruz para o produto RSI, foi uma avaliação mais curta por ser um produto novo e avaliado no momento de seu lançamento. Neste caso, se avalia a documentação existente sobre o produto, a definição dos processos das dimensões Organizacional, Engenharia de Software e Serviços e as características de Qualidade do Produto. Após o Diagnóstico Inicial, a empresa decidiu realizar os ajustes necessários apenas para o nível Bronze, que foi o nível considerado para a avaliação final. Neste caso, também, não houve apoio de consultoria. A Fiocruz já tinha processos definidos e modelados e profissionais com conhecimento de modelagem de processos. O Diagnóstico Inicial foi realizado em novembro de 2016 com duração de 2 dias e a avaliação final em abril 2017 com duração de um dia e meio. A equipe de avaliação foi 
formada no diagnóstico inicial por um avaliador líder e dois avaliadores e na avaliação final por um avaliador líder e um avaliador.

\subsection{Feedback após as avaliações}

Após a finalização das avaliações, foi solicitado às empresas e aos implementadores e avaliadores do QPS que fornecessem um feedback sobre o modelo e as avaliações realizadas, por meio de um questionário. A seguir, estão listados alguns dos feedbacks recebidos.

- "O método de avaliação foi muito bom, com melhorias sensíveis com relação a outros modelos".

- "Percebemos uma preocupação muito forte de que o modelo seja flexível para todos os tipos de empresa que trabalham com produto de software. Essa capacidade de se ajustar aos diferentes modelos de negócio é muito importante para que as empresas consigam extrair o máximo de beneficios e resultados através do modelo".

- "Recebemos com muito entusiasmo esse modelo, que agrega todos os processos que fazem diferença para nós que trabalhamos com produto".

- "Método de avaliação excelente".

Dois implementadores do modelo QPS em empresas com produtos já avaliados observaram:

- "O modelo QPS pode ser visto como um guia para apoiar definição ou melhoria na execução das atividades relacionadas à elaboração $e$ manutenção do produto de software e serviço. Fornece subsídios para apoiar a padronização de atividades, não com sentido "burocrático", mas em facilitar a sua execução. Relativamente fácil de ser implementado, pois contempla e foca nas atividades essenciais à realização e manutenção do produto e do serviço, incluindo aspectos da qualidade e usabilidade do produto. Em função disto, melhora o desempenho do time, facilita a comunicação entre seus componentes $e$ fornece subsídios para a otimização e melhoria dos processos definidos. Pode ser implementado em organização de software, de qualquer tamanho (pequena, média ou grande), que tenha um produto de software disponibilizado ou que venha a ser disponibilizado para clientes. Sendo implementado adequadamente na organização de software, transmite confiança ao implementador na submissão ao Método de Avaliação. As informações contidas no relatório do resultado da avaliação inicial foram de grande valia para conduzir as correções e adequações aos requisitos do modelo".

- "A Avaliação do QPS é singular em relação às demais avaliações de modelos e normas de qualidade disseminadas na indústria. Apesar deste modelo considerar normas como a Série ISO/IEC 33000 em seu processo de avaliação, o fato do QPS possuir uma arquitetura focada em quatro dimensões, amplia-se a percepção do avaliador em relação ao software avaliado. Além disso, seu diferencial destaca-se pelas dimensões focadas na qualidade do produto e não na qualidade do processo. Com isso, as dimensões Organizacional, Engenharia de 
Software, Serviços e Qualidade do Produto descrevem os processos relevantes para a avaliação da qualidade de um produto de software".

A seguir, apresenta-se as observações de avaliadores que participaram das avaliações piloto do QPS:

- "Na avaliação QPS que participei a empresa, entre o diagnóstico inicial e a avaliação final, teve uma melhora enorme em seus processos, o que demostra a assertividade do modelo e do método de avaliação. Achei bom para a empresa ter uma avaliação completa para depois pensar em que nivel se adequa".

- "Minha percepção foi de que a implementação do modelo em empresas que já tem um produto no mercado é bastante simples e rápida. Essas empresas já realizam praticamente todas as atividades previstas no modelo no que se refere à manutenção do produto e ao serviço de atendimento ao cliente.

- Empresas que estejam lançando um produto no mercado pela primeira vez, por não ter experiência podem não ter previsto todos os processos necessários para manutenção do produto e atendimento ao cliente. Neste caso, torna-se necessário um apoio de consultoria na definição de processos. Entretanto, pode estar aí o maior benefício e um elemento diferencial com relação a produtos concorrentes.

- Um aspecto bastante valorizado pelas empresas que tiveram seus produtos avaliados foi o fato de no diagnóstico inicial se fornecer um relatório com todas as questões que precisam ser ajustadas considerando todos os níveis do modelo (Ouro, Prata e Bronze). Isto é possivel pela avaliação QPS ser uma avaliação contínua com resultado em estágios, o que é considerado importante por fornecer um caminho consistente para a melhoria do produto".

- "A empresa, mesmo sem acompanhamento de consultores especializados no modelo QPS, conseguiu compreender e apresentar todas as evidências sem dificuldades. Embora o QPS tenha foco no produto e possua dimensões que o diferenciam dos demais modelos de qualidade com foco em processo, a experiência da equipe da empresa com outros modelos da qualidade auxiliou no entendimento do modelo.

- O feedback que a avaliação QPS é capaz de prover pode contribuir com a reorientação ou confirmação da estratégia geral da empresa, visto que a perspectiva do cliente também é considerada na avaliação.

- O QPS não dissocia a qualidade do produto e a forma como o produto é entregue e percebido pelo cliente. Esta conexão estimula a organização a desenvolver uma visão holística com alinhamento da evolução do produto e serviços associados aos objetivos estratégicos da organização.

- Sob o ponto de vista do avaliador, é necessário quebrar o paradigma dos modelos de qualidade com foco em processos. Nestes modelos cada processo é avaliado de forma isolada, com foco nos seus resultados esperados. No QPS as dimensões se complementam e contribuem para o desenvolvimento do negócio como um todo, com foco no produto".

- "A dimensão Organizacional proporciona um enfoque no produto que nem sempre encontramos quando as empresas implantam mais de um 
modelo. Pude perceber um feedback positivo deste enfoque e acredito que seja de suma importância para empresas de produto. $O$ modelo está mais leve em alguns aspectos de engenharia de software, o que o torna adequado para manutenções de produto, onde o processo precisa ser ágil e leve. A dimensão de Qualidade do Produto possui potencial para evolução e acredito que traz uma atenção especial à qualidade que é muito bem recebida pelas empresas e pode auxiliar no que elas podem buscar para melhorar a qualidade do seu produto. De forma geral, pude notar uma boa recepção do modelo e confio que trará benefícios para as empresas de produto ao orientar seus programas de melhoria”.

\section{Considerações Finais}

Este artigo apresentou o modelo QPS para avaliação da qualidade de produtos de software e o resultado de três avaliações já realizadas. Estas três avaliações foram realizadas como avaliações piloto com o objetivo de avaliar e realizar melhorias no modelo QPS e no método de avaliação.

O feedback recebido das empresas avaliadas e dos implementadores e avaliadores do modelo foi bastante positivo. Dentre as observações pode-se sumarizar:

- O modelo foi considerado fácil de ser implementado, influenciou na melhoria dos processos da organização e o diagnóstico inicial foi bem visto pelas organizações avaliadas. Além disso, a representação contínua do modelo foi considerada adequada.

- A experiência em outros modelos auxilia no entendimento do QPS.

- Em relação à manutenção de produtos, foi percebido que o modelo é aderente às atividades já realizadas por organizações de manutenção e é adequado para este tipo de organização. Além disso, foi destacado que o fato de o modelo prever processos de manutenção e atendimento ao cliente pode representar um diferencial para as organizações.

- O modelo foi considerado flexível para ser utilizado por diferentes empresas que desenvolvem produtos de software e isso possibilita às organizações extrair mais benefícios e resultados de sua implementação.

- O modelo estimula o desenvolvimento de uma visão holística com alinhamento da evolução do produto e serviços associados aos objetivos estratégicos da organização, além disso, considerar a perspectiva do cliente na avaliação pode contribuir para a definição da estratégia geral da empresa.

- Foi reportado que as quatro dimensões do QPS são um diferencial do modelo, por focarem na qualidade do produto e não apenas do processo. Também foi destacado que a forma como as dimensões se relacionam contribui para o desenvolvimento do negócio, com foco no produto. Além disso, foi reforçado que a dimensão Organizacional proporciona um enfoque no produto que não há em outros modelos e que a dimensão de Qualidade de Produto pode auxiliar na busca da melhoria da qualidade do produto pelas organizações.

As três avaliações, realizadas como avaliações piloto, tinham como um de seus objetivos evidenciar a necessidade de realizar melhorias no Modelo QPS e no processo 
e método de avaliação. No momento em que as três avaliações foram realizadas o modelo QPS já estava totalmente definido e o texto descrevendo o modelo foi entregue às empresas. Entretanto, foi sentida a necessidade de se ter uma versão comentada do modelo com explicação dos resultados esperados. $O$ processo de avaliação se mostrou totalmente adequado e não foram apontadas necessidades de ajustes. Para o método de avaliação foram apontadas questões relacionadas à caracterização dos resultados na avaliação final. Por ser uma avaliação de produto foi considerado mais importante caracterizar a situação final do produto, sem valorizar o estágio observado durante o diagnóstico inicial.

Neste momento outras empresas estão se preparando para avaliação de seus produtos no modelo QPS. Foram, também, realizados cursos para formação de implementadores e avaliadores do modelo QPS no Rio de Janeiro, Maceió e Recife que credenciaram avaliadores situados em diversos estados do país. Novos cursos estão planejados para serem realizados ainda em 2017.

O Modelo QPS compõe o conjunto de tecnologias de software oferecidas pelo DELFOS (Observatório da Engenharia do Software Contemporâneo) da COPPE/UFRJ. O modelo QPS para avaliação de produtos de software é o primeiro modelo da família de modelos de qualidade DELFOS. Outros modelos estão sendo definidos para atender a outras situações específicas.

\section{Agradecimentos}

Os autores agradecem a todos que colaboraram na definição e aprimoramento do modelo QPS: Cristina Cerdeiral (UNIRIO), Tayana Conte (UFAM), Reinaldo Cabral (UFAL), Bruno Santana (PROCENGE), Eduardo Cavalcante (PROCENGE), Rafael Figueiredo (Monteiro e Gutierrez Sistemas), Andre Campos (Fiocruz), Pedro Erthal Soares (Fiocruz), Luiz Sergio Plácido da Silva (Softex Recife), Benno Eduardo Albert (Petrobras), Carlos Alberto Simões (Makalu Consultoria Empresarial), Denize Pimenta (UNIRIO), João Alexandre Sartorelli (Petrobras), Peter Peret Lupo (Implementum), Elaine Duarte Nunes (Implementum) e Rachel Vital (COPPE/UFRJ).

\section{Referências}

CMMI Product Team (2010a). CMMI for Development - Version 1.3, Software Engineering Institute, Carnegie Mellon University, Pittsburgh, Pennsylvania, Technical Report CMU/SEI-2010-TR-033.

CMMI Product Team (2010b). CMMI for Services - Version 1.3, Software Engineering Institute, Carnegie Mellon University, Pittsburgh, Pennsylvania, Technical Report CMU/SEI-2010-TR-034.

ISO/IEC (2008a). ISO/IEC 12207 Systems and software engineering- Software life cycle processes, Geneve: ISO.

ISO/IEC (2008b). ISO/IEC 25012:2008 - Software engineering - Software Product Quality Requirements and Evaluation (SQuaRE) - Data Quality Model. Geneve: ISO, 2008.

ISO/IEC (2011). ISO/IEC 20000-1:2011 Information Technology - Service Management. Part 1: Service Management Systems Requirements, Geneve: ISO. 
ISO/IEC (2012). ISO/IEC 20000-2:2012 Information Technology - Service Management. Part 2: Guidance on the application of service management systems, Geneve: ISO.

ISO/IEC (2014). ISO/IEC 25000:2014 - Systems and software engineering - Systems and software Quality Requirements and Evaluation (SQuaRE) - Guide to SQuaRE. Geneve: ISO, 2014.

ISO/IEC (2015). ISO/IEC 33020 - Information Technology - Process Assessment Process Measurement Framework for Assessment of Process Capability. Geneve: ISO, 2015.

Mota, R., Oliveira, K,M. Uma proposta para apoio à identificação de características de qualidade de produtos de software no contexto do modelo QPS, II Workshop de Qualidade de Produto de Software, 2017.

OGC (2011). "ITIL Service Operations" The Stationary Office - TSO. London, UK.

Silveira, B.C.A., Souza, T. S. Atributos e Medidas de Acessibilidade de Software: Reflexões sobre a ISO/IEC 25000, II Workshop de Qualidade de Produto de Software, 2017.

SOFTEX (2015) MPS.BR - Guia Geral MPS de Serviços:2015, outubro 2015. Disponível em www.softex.br/mpsbr, consultado em 29/05/2017.

SOFTEX (2016) MPS.BR - Guia Geral MPS de Software:2016, janeiro 2016. Disponível em www.softex.br/mpsbr, consultado em 29/05/2017.

QPS (2016) Modelo de Referência para Avaliação de Produtos de Software, versão 1.0, outubro 2016. 